\title{
Unsteady wind loads for TMT: Replacing parametric models with CFD
}

\author{
Douglas G. MacMartin*,a,b, and Konstantinos Vogiatzis ${ }^{b}$, \\ ${ }^{a}$ Computing + Mathematical Sciences, California Institute of Technology \\ 1200 E. California Blvd., Pasadena, CA 91125 \\ ${ }^{b}$ TMT Observatory Corp., 1111 S. Arroyo Parkway, Suite 200, Pasadena CA 91105
}

\begin{abstract}
Unsteady wind loads due to turbulence inside the telescope enclosure result in image jitter and higher-order image degradation due to M1 segment motion. Advances in computational fluid dynamics (CFD) allow unsteady simulations of the flow around realistic telescope geometry, in order to compute the unsteady forces due to wind turbulence. These simulations can then be used to understand the characteristics of the wind loads. Previous estimates used a parametric model based on a number of assumptions about the wind characteristics, such as a von Karman spectrum and frozen-flow turbulence across M1, and relied on CFD only to estimate parameters such as mean wind speed and turbulent kinetic energy. Using the CFD-computed forces avoids the need for assumptions regarding the flow.

We discuss here both the loads on the telescope that lead to image jitter, and the spatially-varying force distribution across the primary mirror, using simulations with the Thirty Meter Telescope (TMT) geometry. The amplitude, temporal spectrum, and spatial distribution of wind disturbances are all estimated; these are then used to compute the resulting image motion and degradation. There are several key differences relative to our earlier parametric model. First, the TMT enclosure provides sufficient wind reduction at the top end (near M2) to render the larger cross-sectional structural areas further inside the enclosure (including M1) significant in determining the overall image jitter. Second, the temporal spectrum is not von Karman as the turbulence is not fully developed; this applies both in predicting image jitter and M1 segment motion. And third, for loads on M1, the spatial characteristics are not consistent with propagating a frozen-flow turbulence screen across the mirror: Frozen flow would result in a relationship between temporal frequency content and spatial frequency content that does not hold in the CFD predictions.

Incorporating the new estimates of wind load characteristics into TMT response predictions leads to revised estimates of the response of TMT to wind turbulence, and validates the aerodynamic design of the enclosure.
\end{abstract}

\section{Keywords: Computational Fluid Dynamics (CFD), Wind Loading, Ground Based Telescopes}

\section{INTRODUCTION}

Unsteady wind loads on the telescope structure result in both image jitter and dynamic motion of the primary mirror segments. For the Thirty Meter Telescope (TMT) these forces are minimized by careful enclosure design, and structural design to minimize cross-sectional area. Predicting wind loads during design has been of interest for a long time. ${ }^{1,2}$ Given some description of the wind loads, the optical response can be computed using a finite element model (FEM) of the telescope structure, linear optical model, relevant control loops, and (for predicting segment motion), the dynamics of M1 segments, segment actuators, and actuator servo loops. ${ }^{3,4}$ Previous models of wind loading on the telescope structure for TMT have been based on a parametric model ${ }^{4-6}$ informed by a combination of Gemini data, ${ }^{7,8}$ wind tunnel testing ${ }^{9}$ and CFD. Advances in CFD now allow the unsteady forces on the structure and optics to be computed directly, as a function of external wind speed, orientation of the telescope with respect to the wind, and with enclosure vents either open or closed. The CFD is described in detail in [10], and simulations briefly summarized in Section 2.

The focus here is not on the CFD methods nor the models used for calculating the optical response, but on the relevant characteristics of the flow and resulting forces. There are a number of important differences between the CFD-predicted unsteady forces and those predicted by the parametric model. Sections 3 and 4 describe, respectively, the telescope image jitter and M1 segment motion predicted by the CFD, with particular emphasis on characteristics different from the parametric model.

*Email: macmardg@cds.caltech.edu

Modeling, Systems Engineering, and Project Management for Astronomy $\mathrm{VI}$, edited by George Z. Angeli, Philippe Dierickx, Proc. of SPIE Vol. 9150, 91500Q

(C) 2014 SPIE - CCC code: $0277-786 X / 14 / \$ 18 \cdot$ doi: $10.1117 / 12.2057295$ 

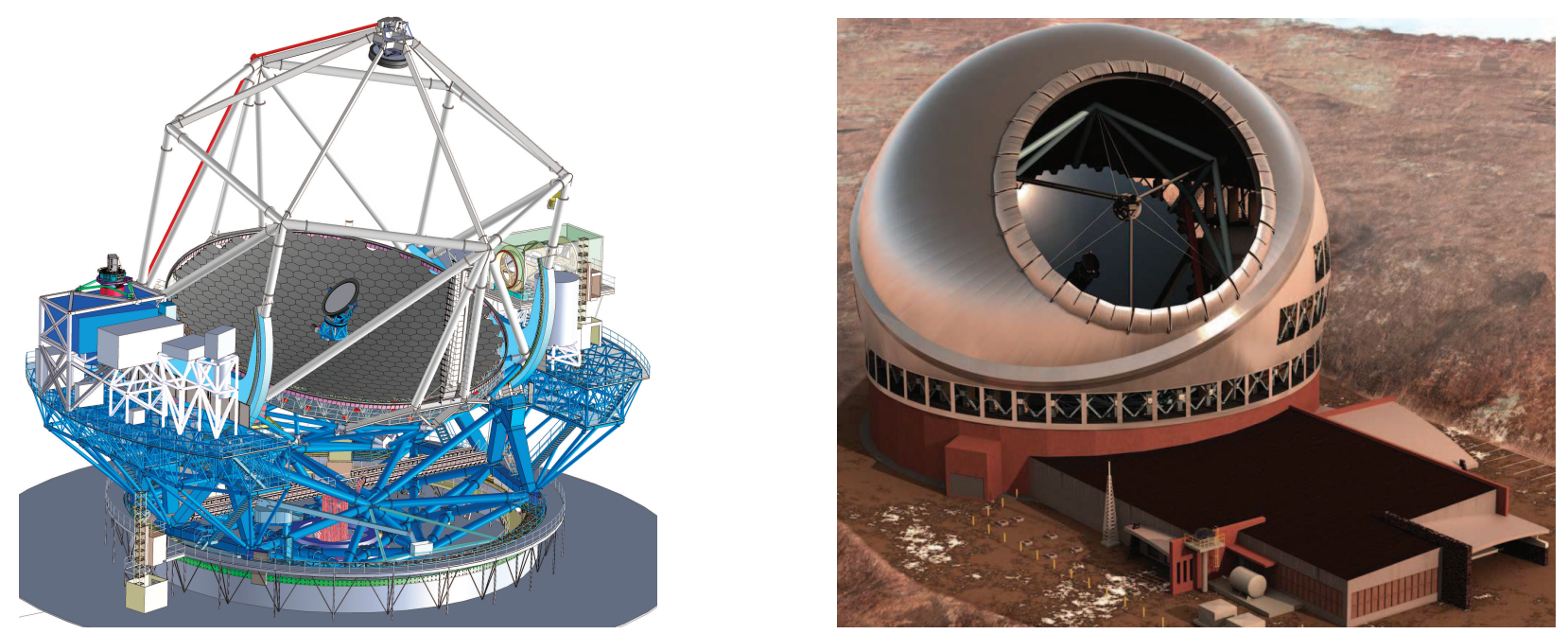

Figure 1. TMT Observatory: telescope (left) and Calotte enclosure (right). For the telescope, the unsteady wind-moment about the elevation axis has been separately calculated for the "top end" (M2 and LGSF), the "spider" (there are 6 support legs connecting the top end to the upper telescope tube), the "tube" (telescope structure below the spider and above M1, and "M1" (primary mirror). Both enclosure vents and aperture flaps are visible at right.

In addition to wind buffeting from unsteady forces, CFD is also used to predict dome and mirror seeing. The relative contribution to telescope performance due to wind-buffeting and seeing can be traded off to some extent by choosing what fraction of the enclosure vent area is opened. At low external wind speeds, a larger open area improves dome and mirror seeing, while at higher external wind speeds, the vents can be closed to reduce wind-buffeting.

\section{CFD SIMULATIONS}

In addition to computing unsteady loads with confidence, one of the fundamental advances that enables simulations across a range of external wind speeds and telescope orientation with respect to the wind is the use of a multi-region CFD model. ${ }^{10}$ This consists of several domains corresponding to all major rotating Observatory components: the telescope elevation structure, azimuth structure, the Calotte enclosure rotating base, vents, cap, and shutter. All of these domains interface with each other and the fixed domain, which includes the fixed enclosure base, facilities building, and local topography. The interface cells can slide relative to each other so that the telescope can point in any direction; this allows the same underlying grid to be used for all calculations. Representative flow patterns for upwind and downwind orientations are shown in Figure 2.

At each orientation, a single 900 second simulation is conducted covering a range of external wind speeds, with a smooth transient between them, and a 150 second section of steady wind-speed section; these steady intervals are used to evaluate the telescope response after transients have settled (see Fig. 3). For computing image jitter, the torque about both the elevation axis and azimuth axis is reported; for several cases, additional simulations report the torque separately for different sub-components to better understand the relative contributions (e.g., Table 1). For computing the motion of the 492 primary mirror segments, pressures are reported on both the front and back surfaces of the mirror at 24 locations on each side of each segment.

Gaps between segments of the primary mirror can in principle allow flow to communicate between the front and back surfaces. However, the gaps are only a few $\mathrm{mm}$, much smaller than the segment thickness, so this primarily affects only the quasi-static pressure distribution. An additional set of simulations were conducted with gaps in the primary mirror to validate that the presence of the gaps was not important in predicting the primary mirror unsteady pressures and resulting segment motion.

\section{IMAGE JITTER}

Modeling of the telescope image jitter due to applied wind loads ${ }^{4}$ uses the finite element model (FEM) of the telescope structure coupled to a linear optical model, and with the main-axes mount control loops closed (roughly $0.5 \mathrm{~Hz}$ bandwidth used here; the final design may have a higher bandwidth) and optical guiding $(0.1 \mathrm{~Hz}$ used here). The telescope response is predicted in the time-domain; see e.g. Fig. 3. 


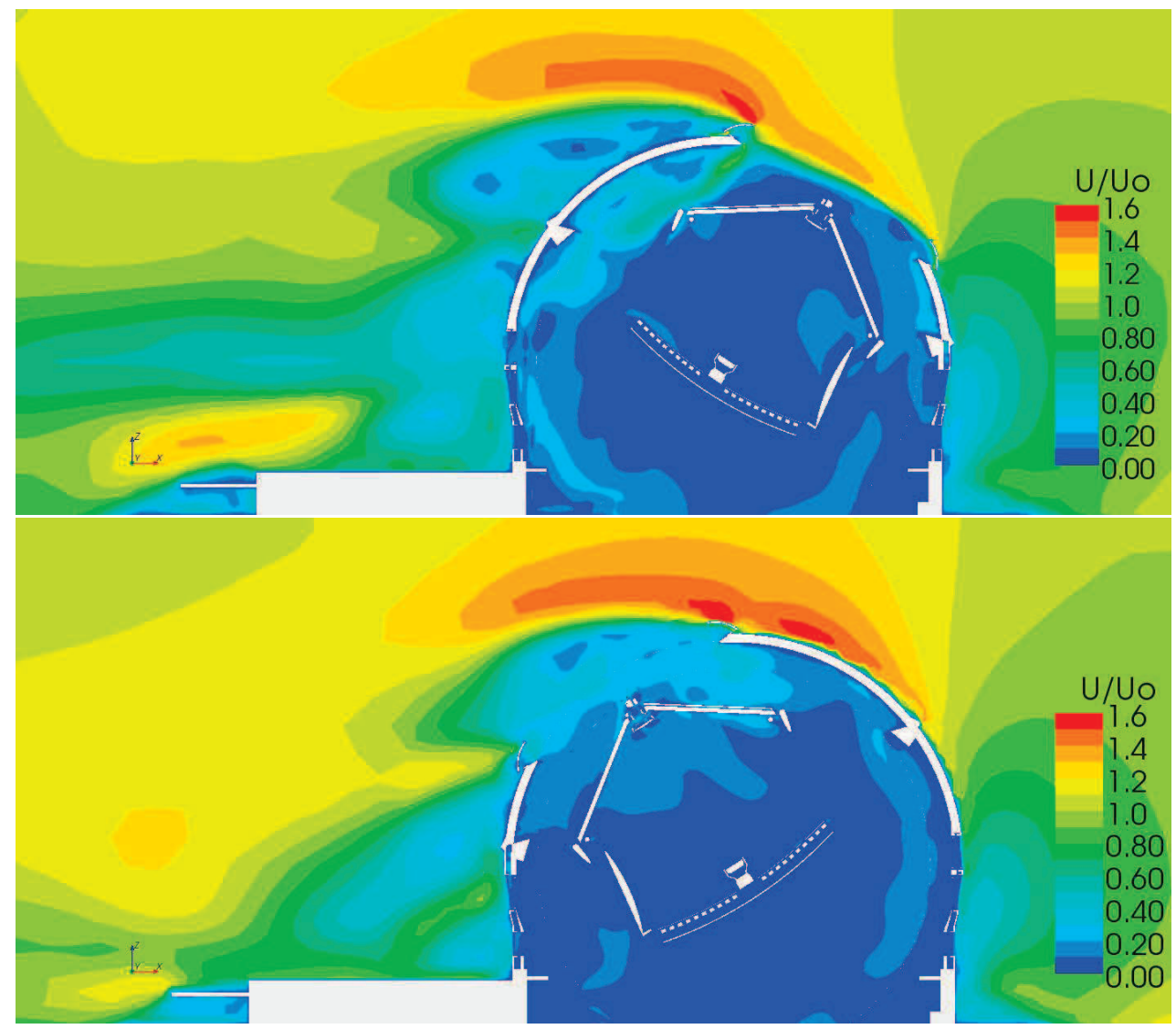

Figure 2. A representative instantaneous velocity field for upwind (top) and downwind (bottom) orientation with respect to the wind, at $33^{\circ}$ zenith angle and with all enclosure vents closed; contours give the ratio of velocity to the upwind reference wind speed. The design of the Calotte enclosure, including aperture flaps, protects the telescope top end from high wind loads in upwind orientations; in this case there is some swirling flow in the enclosure and higher wind speeds around the spider, tube, and primary mirror than at the top end. For downwind orientations the telescope top end and spider are in the recirculation zone of the enclosure wake.

Because the frequency content is low, the dominant contribution to wind-induced image jitter is the rigidbody rotation of the telescope about the elevation axis (and to a lesser extent the azimuth axis) from the finite bandwidth of the mount control system. ${ }^{4}$ Compliance of the telescope structure itself plays a less important role, and the excitation of structural resonances is not important, nor is motion of M2 relative to its support structure due to pressure gradients across its surface. All of these other sources of image motion might become relatively more important if there was a higher bandwidth optical tip/tilt loop, as this guide loop would correct much of the low-frequency MCS contribution. For this reason, only the torque about the main drive axes is used from the CFD in computing the response shown in Fig. 3. Because of the lower moment arm and higher rotational inertia, azimuth-axis rotation is smaller, and the image motion is dominated by rotation about the elevation axis. Quasi-static wind loads are not important as they are rejected by the guide loop.

One of the assumptions that was made in parametric modeling was that the turbulence spectrum was von Karman, appropriate for fully-developed turbulence. This was consistent with both Gemini data and windtunnel tests, however, in both cases the enclosure was more "open" than the Calotte design selected for TMT (see Fig. 1). The temporal spectrum of the net torque about the elevation axis predicted from unsteady CFD is shown in Fig. 4; this is compared with a von Karman spectrum with a $-13 / 3$ final slope (note that the Kolmogorov slope for pressure is $-7 / 3$, but integrating over area means that the slope for force or moments should be $-13 / 3$ ). For a downwind orientation, the von Karman assumption is validated by the CFD. However, for upwind orientations where image jitter is more of a concern, then relative to a von Karman assumption, there can be significant energy in relatively narrow frequency bands. Depending on the wind-speed dependent 

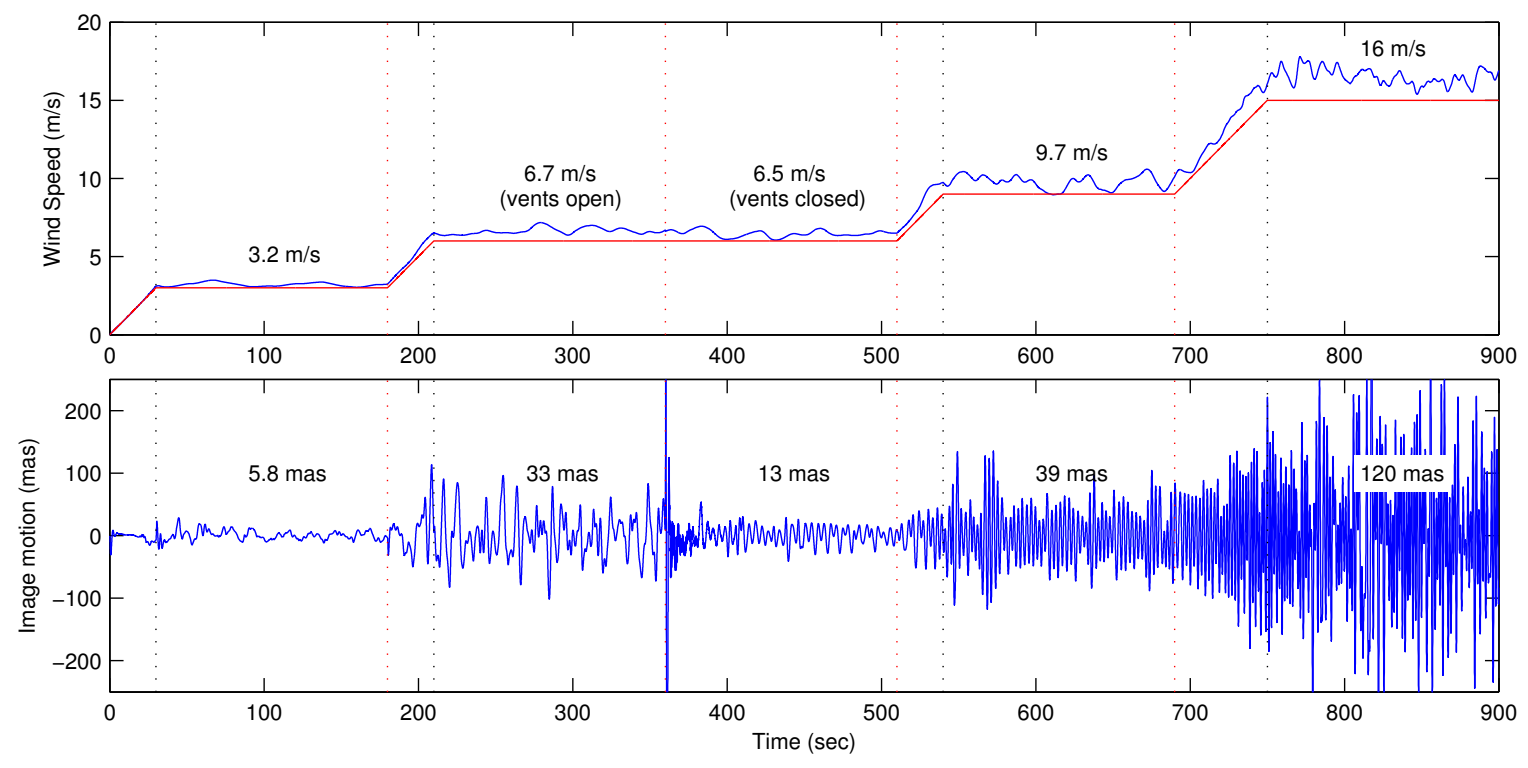

Figure 3. Telescope image jitter about elevation axis due to unsteady CFD-predicted wind loads for increasing external wind speeds for an upwind orientation $\left(33^{\circ}\right.$ zenith angle, and at a $0^{\circ}$ azimuth relative to the wind; facing East). The external wind speed (top panel) is gradually changed in the CFD, with steady time periods (shown by dotted lines) used to evaluate wind response after transients have settled. Enclosure vents are closed at 360 seconds. The smooth velocity curve (red) shows the imposed upwind velocity boundary condition at the end of the domain, while the blue curve is the upwind velocity relevant for comparison with measured site statistics (e.g., for computing probability distributions of image jitter); this is taken $100 \mathrm{~m}$ upwind from the center of the dome, roughly one diameter upstream of the enclosure.
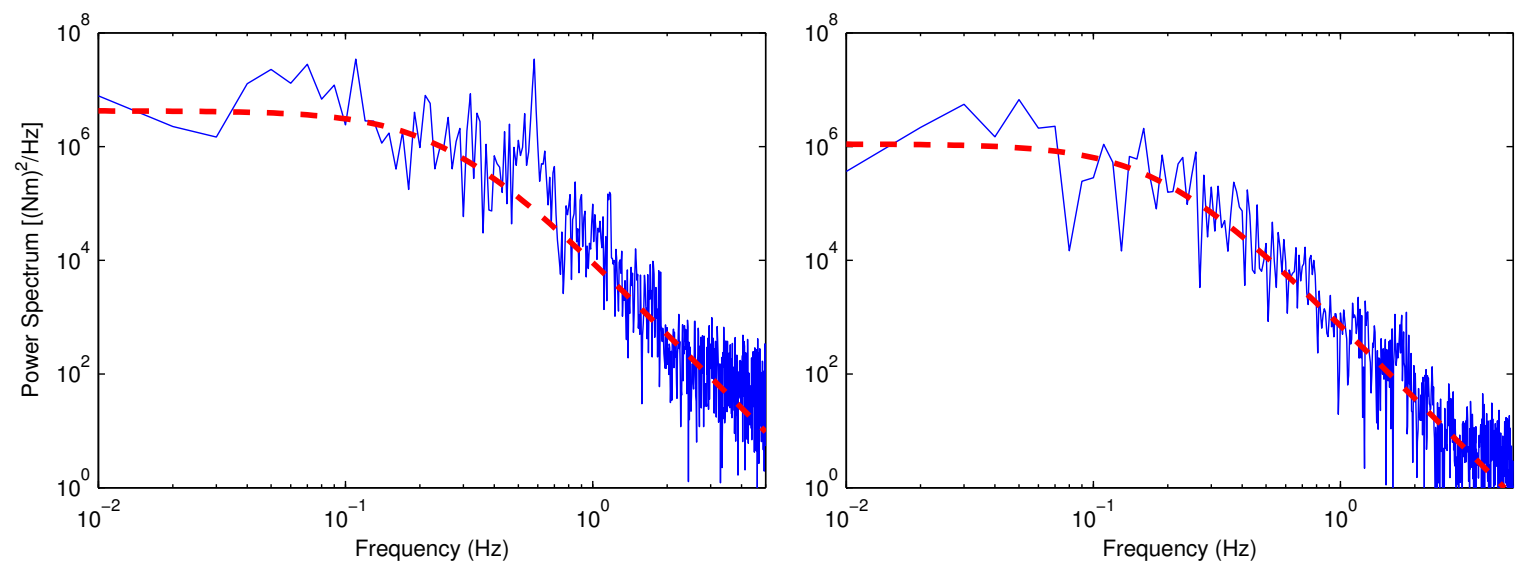

Figure 4. Power spectrum of torque about elevation axis for upwind (left) and downwind (right) orientation with respect to the wind, both at $33^{\circ}$ zenith angle and a $9.7 \mathrm{~m} / \mathrm{s}$ external wind speed. A fit to a von Karman spectrum with a slope of $-13 / 3$ is shown for comparison, using an outer scale of 0.3 and $0.2 \mathrm{~m}$ respectively (corresponding to length scales of $\sim 32$ and $\sim 50 \mathrm{~m}$ ). A von Karman spectrum is a good approximation for downwind torques but the spectrum for the upwind orientation includes significant additional energy near $0.6 \mathrm{~Hz}$ that accounts for roughly half of the image jitter variance.

frequency at which these occur, this can either increase or decrease the resulting image jitter relative to a von Karman spectrum with similar rms torque. The mount control bandwidth is roughly $0.5 \mathrm{~Hz}$; disturbances well below this frequency are adequately rejected, but the peak of the torque-rejection curve is at frequencies near the control bandwidth.

Parametric models for TMT assumed that, at least for high external wind speed where image jitter is a potential concern, that the combination of larger moment arm and higher wind speeds near the top of the telescope (M2 and laser guide star facility, LGSF) would result in larger unsteady torques than the lower wind speeds deeper inside the telescope enclosure, despite the difference in cross-sectional area. At low external wind 


\begin{tabular}{l|cc|ccc|} 
& $\begin{array}{c}3.2 \\
\text { open }\end{array}$ & $\begin{array}{c}6.7 \\
\text { open }\end{array}$ & $\begin{array}{c}6.5 \\
\text { closed }\end{array}$ & $\begin{array}{c}9.7 \\
\text { closed }\end{array}$ & $\begin{array}{c}16.4 \\
\text { closed }\end{array}$ \\
\hline Top end & 1 & 3 & 4 & 15 & 34 \\
Spider & 1 & 4 & 3 & 9 & 24 \\
Tube & 1 & 12 & $\mathbf{9}$ & $\mathbf{2 6}$ & $\mathbf{6 2}$ \\
M1 cell & $\mathbf{5}$ & $\mathbf{3 3}$ & 4 & 15 & 49 \\
\hline Total & 6 & 35 & 14 & 42 & 103
\end{tabular}

\begin{tabular}{l|cc|ccc|} 
& $\begin{array}{c}3.2 \\
\text { open }\end{array}$ & $\begin{array}{c}6.7 \\
\text { open }\end{array}$ & $\begin{array}{c}6.5 \\
\text { closed }\end{array}$ & $\begin{array}{c}9.7 \\
\text { closed }\end{array}$ & $\begin{array}{c}16.4 \\
\text { closed }\end{array}$ \\
\hline Top end & 1 & 7 & $<1$ & $\mathbf{4}$ & $\mathbf{2 2}$ \\
Spider & 1 & 7 & $<1$ & $\mathbf{5}$ & $\mathbf{2 4}$ \\
Tube & 2 & 15 & $<1$ & 1 & 5 \\
M1 cell & $\mathbf{5}$ & $\mathbf{2 1}$ & 1 & 2 & 7 \\
\hline Total & 7 & 28 & 1 & 8 & 45
\end{tabular}

Table 1. Predicted image jitter (in mas) due to torque on different components of the telescope (see Fig. 1), for a $33^{\circ}$ zenith angle and upwind orientation with respect to wind (left) and downwind (right). If vents are open, then the M1 cell is the dominant contributor (not surprisingly) for either upwind or downwind orientations. If vents are closed then the largest contribution in upwind orientations is from the tube, but contributions from all components are relevant, while for downwind orientations it is only the top end and spider that provide significant contributions. The final "total" line may not be the quadrature sum of individual components due to partial correlations or anti-correlations between torques on different components.

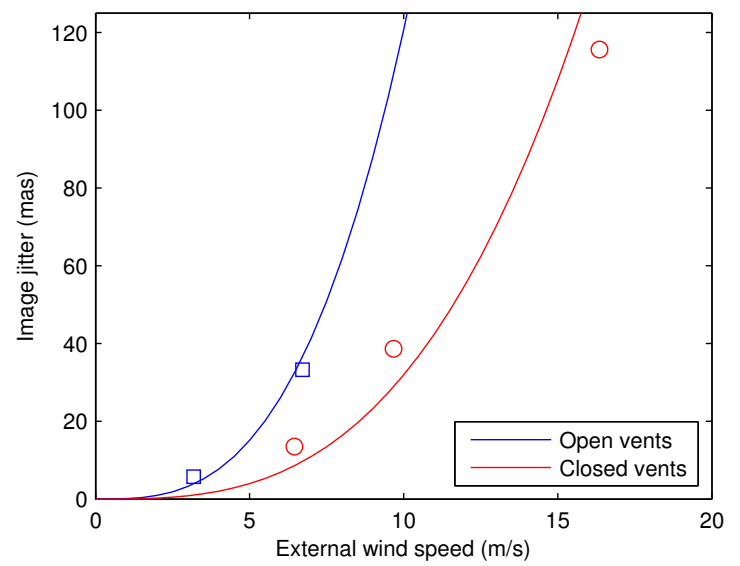

\begin{tabular}{|c|c|c|c|c|}
\hline Zenith & \multicolumn{4}{|c|}{ Orientation } \\
Angle & $\mathrm{E}$ & $\mathrm{S}$ & $\mathrm{W}$ & $\mathrm{N}$ \\
\hline 1 & \multicolumn{5}{|c|}{12.3} \\
33 & 33.3 & 24.3 & 25.6 & 6.4 \\
65 & 95.2 & 29.5 & 27.6 & 33.5 \\
\hline \hline 1 & \multicolumn{5}{|c|}{5.5} \\
33 & 13.5 & 1.2 & 3.7 & 4.7 \\
65 & 3.1 & 2.1 & 5.6 & 2.0 \\
\hline
\end{tabular}

Figure 5. Predicted image jitter (in mas) as a function of external wind speed (left) and orientation (right). Dependence on wind speed is shown for a $33^{\circ}$ zenith angle and upwind orientation with respect to the wind $\left(0^{\circ}\right.$ relative azimuth), and with vents open and closed. A fit to a $U^{3}$ dependence is also shown. The dependence on orientation is shown for a $6.5 \mathrm{~m} / \mathrm{s}$ wind speed for both vents open (upper rows) and vents closed (lower rows). The wind is from the East, so "E" is upwind and "W" downwind.

speeds, then vents in the telescope enlosure would be open to reduce dome and mirror seeing; this clearly would increase wind speeds near M1 relative to those at the top end, however, this is not the regime in which telescope image jitter matters. While the telescope top end cross-sectional area may indeed be the dominant contributor to torque for many enclosure and telescope designs, careful CFD-informed optimization of the TMT Calotte dome has led to sufficient shielding of the telescope top end so that this is no longer a good assumption for TMT; see Fig. 2. Furthermore, swirling flow in the telescope enclosure results in potentially larger forces near the periphery of M1, resulting in higher than expected torque contributions. The relative contribution to TMT image jitter for different telescope components is shown in Table 1.

Relative to previous TMT analyses, and for upwind orientations in particular, we thus have (i) different relative frequency content that can increase or decrease predicted image jitter, and (ii) lower wind forces near the top end but larger contributions from M1 and the lower telescope structure, where cross-sectional areas are much higher. The top end cross-sectional area has been increased relative to earlier designs to allow for 6 support legs rather than 3 (for earthquake resistance). The combination of all of these factors results in image jitter predictions reasonably consistent with past predictions. Results are shown in Figure 5 as a function of orientation and external wind speed, vents open and closed. Note that the image jitter scales approximately with the cube of external velocity: forces scale with $U^{2}$, but the frequency content also scales with $U$, resulting in poorer rejection by the mount control system at higher wind speeds. The PSSN impact due to image jitter thus scales with $U^{6}$, making the ability to close vents at high external wind speeds essential. 

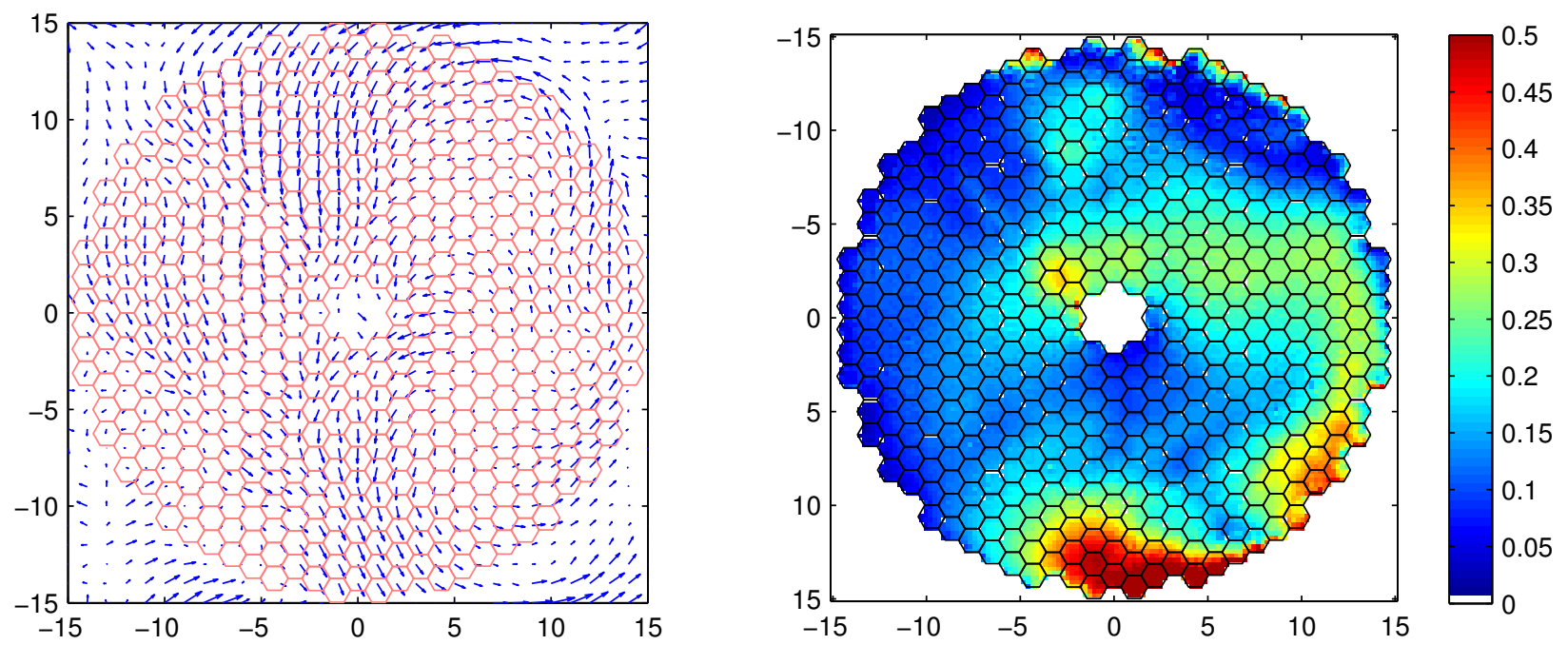

Figure 6. Mean wind speed velocity over M1 for an upwind case with closed vents (left), and rms differential pressure distribution for the same conditions (right). Frozen turbulence is clearly a poor assumption.

\section{PRIMARY MIRROR SEGMENT MOTION}

The mean wind speed over the primary mirror, and the rms unsteady pressure, are shown for a closed-vent case in Figure 6; it is clear that the frozen turbulence assumption used in earlier analyses is not likely to be a good approximation to the complicated and non-uniform flow patterns across M1.

The M1 response is computed from the last 20 seconds of each steady wind speed section in Fig. 3 . The normal force and relevant moments on each segment of the primary mirror are computed from the differential pressure between the front and back surfaces. The response of each segment to the forces and moments is computed from a dynamic model that includes the segment support quasi-static compliance (but not the resonant behaviour), mirror cell and telescope structure compliance (not resonant behaviour), the primary mirror actuator dynamics and actuator servo control law, and finally, the global control loop that uses edge sensor feedback to correct motion other than piston, tip, tilt, and focus at a roughly $1 \mathrm{~Hz}$ bandwidth. A focus bandwidth of $0.5 \mathrm{~Hz}$ is assumed here, and global piston, tip and tilt are unobservable. Resonances of the segment support structure and telescope structure are at frequencies where the wind energy is negligible (see Fig. 7) and can be ignored.

TMT has selected "soft" voice-coil actuators to position the M1 segments, these require a servo loop to provide quasi-static stiffness; the achievable bandwidth for TMT is roughly $8 \mathrm{~Hz}^{11}$ One advantage to these actuators is that they serve to isolate the primary mirror from mirror cell vibration above the frequency of the servo bandwidth. However, one potential drawback is that while the static stiffness is high, the dynamic stiffness to unsteady wind loads is lower than it would be for a hard actuator - the trade-off between these two performance concerns was a key driver of the design decision, and thus understanding wind loads on M1 is crucial. The M1 wind response is determined largely by properties of the actuator servo law, and the integral gain of the servo PID in particular. The temporal frequency content of the wind is important in determining the response because at lower frequencies there is both higher stiffness of the actuator servo loop and higher gain of the edge-sensor feedback loop.

After predicting M1 segment motion, the optical performance can be computed; the adaptive-optics residual can be approximated using spatial filtering, and the PSSN calculated for seeing-limited observations. Because the wind loads are predominantly at low frequency, and correlation lengths are at least multiple segments across, the residual AO-corrected wavefront errors are small, and it is the seeing-limited performance that is the primary concern. The optical performance depends not only on the magnitude of segment dynamic motion, but also on the spatial length-scale. To understand differences between the parametric model and CFD, we thus focus on the magnitude of the rms pressure, the temporal frequency content, the spatial frequency content, and the relationship between these last two.

While the rms pressure in the parametric model was calibrated with CFD, the outer-scale of turbulence was unknown. At Gemini Observatory, the enclosure vents, the primary mirror, and the aperture are all of roughly similar size. As a result, the turbulence length-scale inferred from that data does not immediately help in scaling 

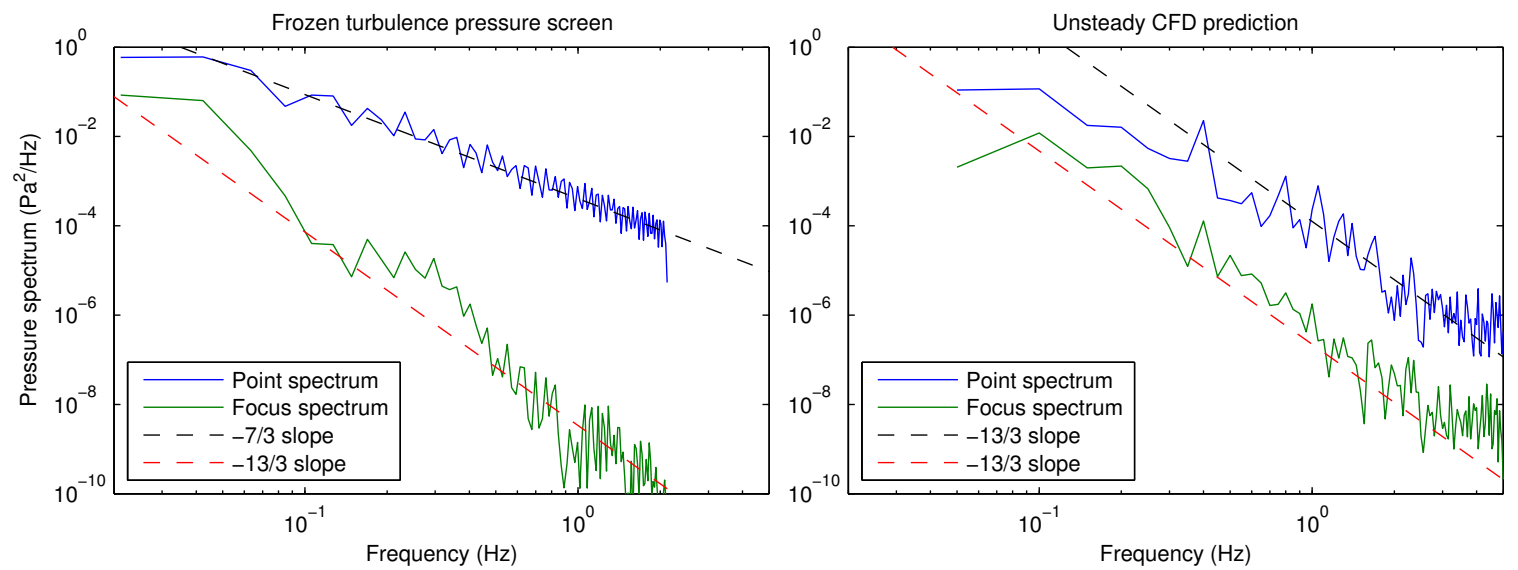

Figure 7. Pressure spectrum at a single point (representative of high spatial-frequency content), and for the projection of the pressure field onto focus (the lowest relevant spatial-frequency), predicted either from a von Karman frozen-flow pressure field over M1 (left) or from CFD (right); the dashed lines indicate slopes. The spectrum at any point based on CFD has a faster decrease in power with frequency than the von Karman assumption. The CFD-based flow field also shows no change in characteristic slope with spatial-frequency, inconsistent with a frozen-flow assumption.

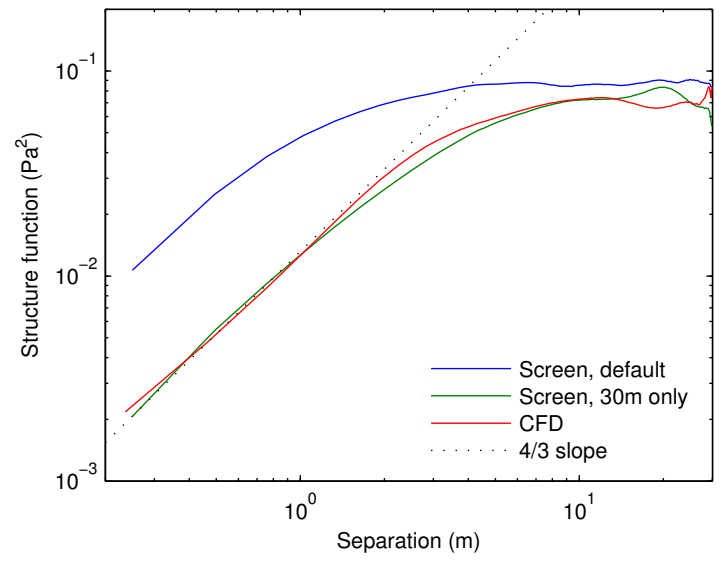

Figure 8. Structure function for unsteady pressures across M1 computed either from CFD or from a von Karman frozen-flow hypothesis; the latter is shown for both a $30 \mathrm{~m}$ outer scale and for a mix of $5 \mathrm{~m}$ and $30 \mathrm{~m}$ scales used in earlier analyses. The CFD spatial correlations are consistent with von Karman turbulence with a $30 \mathrm{~m}$ outer scale, but the temporal characteristics differ.

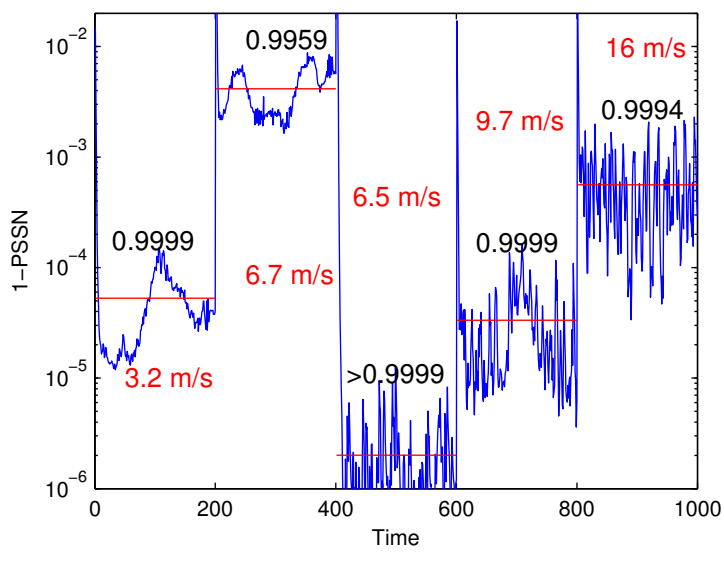

Figure 9. Seeing-limited performance (PSSN) versus time; time is not contiguous in this plot but rather each interval corresponds to the final 20 seconds of the steady windspeed intervals in Fig. 3. The first two cases at 3.2 and $6.7 \mathrm{~m} / \mathrm{s}$ are with vents open, the remaining cases are with vents closed, all at $33^{\circ}$ zenith angle facing upwind.

to TMT. Prior analysis conservatively assumed that turbulence would have a mix of outer scales due to the aperture $(\sim 30 \mathrm{~m})$ and vent height $(\sim 5 \mathrm{~m})$, with the ratio determined by the fraction of wind loads that would have been present had all vents been closed. The structure function from CFD shows dominant length-scales of order $30 \mathrm{~m}$, not $5 \mathrm{~m}$ (Fig. 8), and images of the predicted flow suggest that it is the interaction of large-scale flow with the primary mirror that is the dominant source of turbulence rather than from flow passing through the enclosure vents.

While the temporal power spectrum of torque disturbances (Fig. 4) is not too different from von Karman, the spectrum of M1 pressure descreases much faster with increasing frequency than von Karman (Fig. 7). (Indeed, there can be large-scale separation over M1; it is no surprise that turbulence is not fully developed).

Frozen turbulence also results in a relationship between spatial and temporal frequency content. With frozen turbulence, higher temporal frequencies correspond to shorter spatial length-scales, and conversely, there is higher temporal frequency content in disturbances at short length scales. This is not true in the CFD predictions (Fig. 7), where all length-scales change with comparable temporal spectrum; this is consistent, for example, with a fixed 


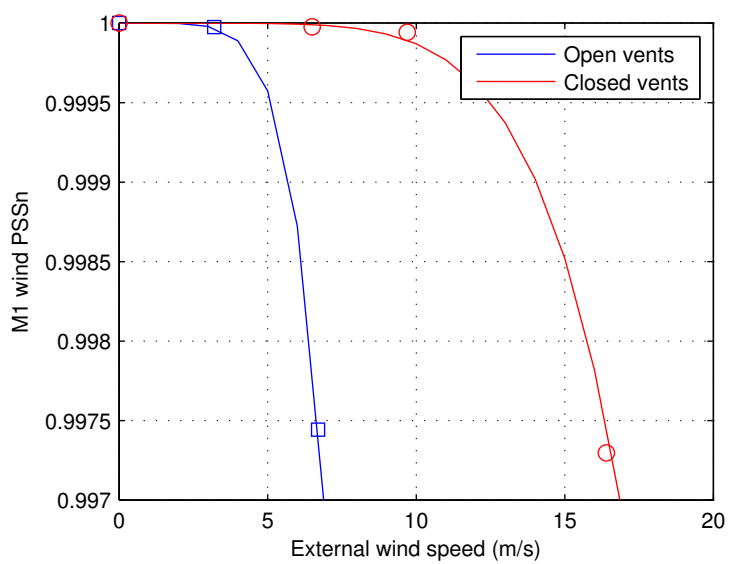

\begin{tabular}{|c|c|c|c|c|}
\hline Zenith & \multicolumn{4}{|c|}{ Orientation } \\
Angle & $\mathrm{E}$ & $\mathrm{S}$ & $\mathrm{W}$ & $\mathrm{N}$ \\
\hline 1 & \multicolumn{5}{|c|}{0.9993} \\
33 & 0.9957 & 0.9942 & 0.9999 & 0.9999 \\
65 & 0.9974 & 0.9975 & $>0.9999$ & 0.9966 \\
\hline \hline 1 & \multicolumn{5}{|c}{0.9893} \\
33 & 0.9994 & 0.9999 & 0.9999 & 0.9900 \\
65 & 0.9995 & 0.9999 & 0.9997 & 0.9999 \\
\hline
\end{tabular}

Figure 10. M1 performance predictions (PSSN) as a function of external wind speed (left) and orientation (right). Dependence on wind speed is shown for a $33^{\circ}$ zenith angle and upwind orientation with respect to the wind $\left(0^{\circ}\right.$ relative azimuth), and with vents open and closed. A fit to a $U^{6}$ dependence is also shown. The dependence on orientation is shown for vents open at a $6.5 \mathrm{~m} / \mathrm{s}$ wind speed (upper rows) and vents closed at $16.4 \mathrm{~m} / \mathrm{s}$ (lower rows; at lower wind speeds the vents-closed PSSN is almost indistinguishable from unity). The wind is from the East, so "E" is upwind and "W" downwind.

spatial pattern that has a time-varying amplitude. Because it is the higher temporal frequencies that are poorly corrected by control (both by the actuator servo and the global loop), while the higher spatial frequencies have more significant optical performance impact for the same rms motion, this difference in character between the parametric assumption and the CFD is extremely significant. With frozen turbulence, the high spatial frequencies are poorly controlled, but with CFD-predicted loads they are as well-corrected by the primary mirror control system as the low spatial-frequencies. Between this difference and the $30 \mathrm{~m}$ rather than $5 \mathrm{~m}$ characteristic lengthscale, the optical consequences of wind-induced pressure fluctuations across M1 predicted by CFD are small.

The performance is shown in Figures 9 and 10. For comparison, with the same control bandwidths, the parametric model used earlier predicts a PSSN of 0.996 for median conditions. Nonetheless, while the performance impact due to wind-induced M1 segment dynamic motion is much smaller than past predictoins, the $U^{6}$ scaling of PSSN impact with velocity makes it essential to be able to close enclosure vents at high external wind speeds.

\section{CONCLUSIONS}

Forces predicted by unsteady CFD are used to predict both the telescope image jitter and M1 dynamic segment motion for TMT, and the relevant characteristics of the forces compared with previous parametric models for telescope wind loading.

For image motion, the resulting predictions are similar to past predictions, however, for upwind orientations, the primary mirror and lower telescope structure are comparatively larger contributors to the net torque than top-end forces. This is partly due to lower top-end wind speeds and higher wind-speeds around M1. The turbulence spectrum is also not von Karman, and can have narrowband spectral components that increase the image jitter if they occur close to the bandwidth of the mount control system where there is poor torque rejection.

For M1, the optical performance resulting from CFD-predicted unsteady pressures is significantly lower than parametric models that assumed von Karman frozen turbulence. One factor is that prior analysis intentionally made a conservative assumption on outer scale. A second is the lower frequency content from non-fully developed turbulence. The frozen turbulence assumption enforces a relationship between temporal and spatial frequencies, so that disturbances at shorter length-scales have higher frequency content that is in turn more poorly rejected by the M1 control system. This relationship does not hold in CFD predictions; the optically-important shorterlength-scale disturbances also have low temporal frequency content and are readily correctable by M1CS, leading to small residual performance implications.

\section{Acknowledgments}

The TMT Project gratefully acknowledges the support of the TMT collaborating institutions. They are the Association of Canadian Universities for Research in Astronomy (ACURA), the California Institute of Technology, the University of California, the National Astronomical Observatory of Japan, the National Astronomical 
Observatories of China and their consortium partners, and the Department of Science and Technology of India and their supported institutes. This work was supported as well by the Gordon and Betty Moore Foundation, the Canada Foundation for Innovation, the Ontario Ministry of Research and Innovation, the National Research Council of Canada, the Natural Sciences and Engineering Research Council of Canada, the British Columbia Knowledge Development Fund, the Association of Universities for Research in Astronomy (AURA), the U.S. National Science Foundation and the National Institutes of Natural Sciences of Japan.

\section{REFERENCES}

[1] Forbes, F. and Gabor, G., "Wind Loading of Large Astronomical Telescopes," SPIE 332 Advanced Technology Telescopes, 1982, pp. 198-205.

[2] Ravensbergen, M., "Main axes servo systems of the VLT," SPIE 2199 Advanced Technology Optical Telescopes V (Stepp, L., ed.), 1994, pp. 997-1005.

[3] MacMynowski, D. G., Thompson, P. M., Shelton, J. C., Roberts, Jr., L. C., Colavita, M. M., and Sirota, M. J., "Control System Modeling for the Thirty Meter Telescope Primary Mirror," Proc. SPIE 8336, 2011.

[4] MacMynowski, D. G. and Andersen, T., "Wind buffeting of large telescopes," Applied Optics, Vol. 49, No. 4, pp. 625636, 2010.

[5] MacMynowski, D. G., Vogiatzis, K., Angeli, G. Z., Fitzsimmons, J., and Nelson, J., "Wind Loads on Ground-Based Telescopes," Applied Optics, Vol. 45, No. 30, pp. 7912-7923, 2006.

[6] MacMynowski, D. G., Colavita, M. M., Skidmore, W., and Vogiatzis, K., "Primary mirror dynamic disturbance models for TMT: Vibration and wind," Proc. SPIE 7738, 2010.

[7] Cho, M. K., Stepp, L. M., Angeli, G. Z., and Smith, D. R., "Wind loading of large telescopes," Large Ground-Based Telescopes (Oschmann, and Stepp, eds.), 2002, pp. 352-367. Proc. SPIE 4837.

[8] Angeli, G. Z., Cho, M. K., Sheehan, M., and Stepp, L. M., "Characterization of Wind Loading of Telescopes," Proc. SPIE Vol. 4757; Workshop on Integrated Modeling of Telescopes (Andersen, T., ed.), 2002, pp. 72-83.

[9] Pottebaum, T. and MacMynowski, D. G., "Buffeting of large telescopes: Wind tunnel measurements of the flow inside a generic enclosure," J. Fluids and Structures, Vol. 22, pp. 3-19, 2006.

[10] Vogiatzis, K., "Transient aero-thermal simulations for TMT," Proc. SPIE 9150, 2014.

[11] MacMartin, D. G., Thompson, P., Colavita, M. M., and Sirota, M. J., "Dynamic analysis of the active-controlled segmented mirror of the Thirty Meter Telescope," IEEE Trans. Control Sys. Tech., 2013. 